\title{
Cyclosporin in atopic dermatitis: a multicentre placebo-controlled study
}

\author{
TH.VAN JOOST, F.HEULE, M.KORSTANJE,* M.J.T.B.VAN DEN BROEK, $\dagger$ \\ H.J.STENVELD $\ddagger$ AND W.A.VAN VLOTEN§ \\ Department of Dermatology. University Hospital Rotterdam-Dijkzigt, Rotterdam. The Netherlands \\ *Department of Dermatology. University Hospital, Maastricht, The Netherlands \\ †Department of Dermatology. Elkerliek Hospital. Helmond, The Netherlands \\ $\ddagger$ Department of Dermatology. University Hospital, Free University. Amsterdam. The Netherlands \\ \$Department of Dermatology. University Hospital, Utrecht. The Netherlands
}

Accepted for publication 1 November 1993

Summary The efficacy of cyclosporin (Sandimmun ${ }^{3}$ ) given in a daily dose of $5 \mathrm{mg} / \mathrm{kg}$ for 6 weeks in severe atopic dermatitis was confirmed in this double-blind, placebo-controlled, short-term study. Of the 46 patients included in the study, 23 were randomized to receive cyclosporin and 23 to receive placebo.

Four of the 23 patients ( $17 \%$ ) on cyclosporin, and 14 of the 23 patients $(61 \%)$ who received placebo, discontinued the trial because of inefficacy. All patients who discontinued the trial were assessed following the principle of 'intention to treat'. Compared with the baseline, the mean scores for disease severity [6-area, total body severity assessment (TBSA)] improved by $55 \%$, and the mean scores for extent of disease [rule-of-nines area assessment (RoNAA)] improved by $40 \%$. in patients treated with cyclosporin. Nine of the patients who received cyclosporin and completed the study $(n=14)$ had an individual reduction of disease severity (TBSA) of $75 \%$ or more, and in three patients this reduction was nearly $100 \%$. In the placebo group, a mean worsening of disease severity (4\%) and of extent of the disease (25\%), compared with the baseline, was observed at week 6 . Patients' and investigators' mean scores for the overall efficacy were similar, and showed a statistically significant difference in favour of cyclosporin.

Two patients on cyclosporin developed hypertension during therapy, and one of these withdrew from the study. At the end of the trial, no statistically significant differences in the systolic or diastolic blood pressures were observed between the two groups. In the cyclosporin group, the increases in the values of serum creatinine and bilirubin at week 6 , compared with the respective values at the baseline, were statistically significantly different from those in the placebo group, but all values normalized in the post-treatment period.

Cyclosporin can be a safe and very effective treatment in episodes of severe atopic dermatitis, provided that the recommended guidelines for its administration are strictly observed.

Atopic dermatitis is a disease with a high prevalence in the general population. It can cause affected individuals a great deal of physical and psychological stress, and may adversely affect work, recreation and relationships. The accompanying pruritus also affects mood and sleep, and thus seriously disturbs the quality of life of the patient. ${ }^{1}$ Some patients are resistant to, or develop sideeffects from, available therapies, such as topical agents or systemic steroids. Consequently, there is a require-

Correspondence: Professor Dr Th.van Joost, Department of DermatoVenereology. Academic Hospital Rotterdam-Dijkzigt, Dr. Molewaterplein 40, 3015 GD Rotterdam. The Netherlands. ment for an effective alternative therapy for the management of severe atopic dermatitis.

Van Joost et al. reported two patients with severe atopic dermatitis who improved considerably within 10 days of receiving cyclosporin therapy in a dose of $5 \mathrm{mg} /$ $\mathrm{kg} /$ day. ${ }^{2}$ The efficacy of cyclosporin in atopic dermatitis was subsequently confirmed in several open studies, ${ }^{3-10}$ and in placebo-controlled crossover studies. ${ }^{11.12}$ Topical application of cyclosporin in atopic dermatitis was reported as being ineffective. ${ }^{13}$

The aim of the present multicentre, double-blind. randomized and placebo-controlled parallel-group study was to determine the efficacy, safety and tolerability of cyclosporin in atopic dermatitis. 
Table 1. Scores of disease severity [6-area total body severity assessment (TBSA)]. Intention to treat principle

\begin{tabular}{|c|c|c|c|c|c|c|}
\hline 0 & 1 & 2 & 3 & 4 & 6 & $\begin{array}{c}\text { Shift }{ }^{*} \\
\text { (week 0-week 6) }\end{array}$ \\
\hline \multicolumn{7}{|c|}{ Cyclosporin } \\
\hline $57 \pm 21$ & $46 \pm 21$ & $31 \pm 21$ & $27 \pm 20$ & $27 \pm 22$ & $25 \pm 23$ & -55 \\
\hline 29-102 & $18-101$ & $4-78$ & $4-67$ & 4-69 & $0-69$ & \\
\hline \multicolumn{7}{|c|}{ Placebo } \\
\hline $54 \pm 22$ & $52 \pm 14$ & $50 \pm 13$ & $50 \pm 14$ & $47 \pm 16$ & $48 \pm 16$ & +4 \\
\hline $26-97$ & $32-83$ & $24-72$ & $31-82$ & $20-82$ & $24-82$ & \\
\hline $0.5 t$ & & & & & & $0.0002 \div$ \\
\hline
\end{tabular}

$n=46$. Cyclosporin, 23 patients: placebo, 23 patients. SD, standard deviation.

* Shift in the percentage of mean values.

$\dagger P$-value (not statistically significant) for the difference between the cyclosporin group and the placebo group at week 0 .

$\ddagger P$-value (statistically significant) for the difference in the mean shifts between the cyclosporin group and the placebo group (week 0 -week 6 ).

\section{Methods}

Cyclosporin was administered in a dose of $5 \mathrm{mg} / \mathrm{kg} /$ day for 6 weeks, to patients suffering from severe, longstanding atopic dermatitis which was resistant to other therapies, and was causing the patients significant suffering and disability. Adult patients, aged between 17 and 68 years, who were suffering from severe refractory atopic dermatitis, were enrolled in the study. At each centre, one investigator was responsible for the clinical assessments and recording of laboratory abnormalities. Approval for the study was obtained from the ethical committees in each of the participating centres. Informed consent was obtained from the patients, who were recruited in the out-patient departments at five centres in the Netherlands. All patients had dermatitis which was resistant to previous therapies, including steroid creams or ointments, and/or PUVA therapy. and/or systemic steroids.

The diagnosis of atopic dermatitis was made according to the criteria of Hanifin and Rajka. ${ }^{14}$ The mean scores (and range) of disease severity and disease extent are summarized in Tables 1 and 2 .

Patients were excluded if they had abnormal renal or hepatic function, hyperkalaemia, hypertension (including drug-controlled hypertension), a history of malignant disease, acute uncontrolled infections, epilepsy, malabsorption syndrome, drug or alcohol abuse, and if

Table 2. Scores of disease extent [rule-of-nines area assessment (RoNAA)]

\begin{tabular}{|c|c|c|c|c|c|c|c|}
\hline & \multicolumn{6}{|c|}{ Week } & \multirow{2}{*}{$\begin{array}{c}\text { Shift* } \\
\text { (week 0-week 6) }\end{array}$} \\
\hline & 0 & 1 & 2 & 3 & 4 & 6 & \\
\hline \multicolumn{8}{|l|}{ Cyclosporin } \\
\hline Mean $\pm \mathrm{SD}$ & $59 \cdot 3 \pm 21 \cdot 8$ & $52 \cdot 3 \pm 23 \cdot 8$ & $36 \cdot 5 \pm 22 \cdot 2$ & $35 \cdot 6 \pm 23 \cdot 2$ & $34 \cdot 0 \pm 23 \cdot 4$ & $33 \cdot 1 \pm 26 \cdot 0$ & -40 \\
\hline Range & $15-100$ & $15-100$ & $6-100$ & $3-99$ & $3-87$ & $0-87$ & \\
\hline \multicolumn{8}{|l|}{ Placebo } \\
\hline Mean \pm SD & $55 \cdot 4 \pm 20 \cdot 7$ & $56 \cdot 5 \pm 18 \cdot 4$ & $59 \cdot 5 \pm 22 \cdot 9$ & $57 \cdot 5 \pm 23 \cdot 6$ & $59 \cdot 3 \pm 23 \cdot 2$ & $59 \cdot 7 \pm 23 \cdot 6$ & +25 \\
\hline Range & $18-100$ & $18-100$ & $27-100$ & $33-100$ & $24-100$ & $33-100$ & \\
\hline$P$-value (Wilcoxon test) & $0 \cdot 7 \dagger$ & & & & & & $0 \cdot 0009 \div$ \\
\hline
\end{tabular}

$n=46$. Cyclosporin, 23 patients; placebo, 23 patients. SD, standard deviation.

- Shift in the percentage of mean values.

$\dagger P$-value (not statistically significant) for the differences between the cyclosporin group and the placebo group at week 0 .

$\ddagger P$-value (statistically significant) for the difference in the mean shifts between the cyclosporin group and the placebo group (week 0 -week 6 ). 


\begin{tabular}{|c|c|c|}
\hline & $\begin{array}{l}\text { Cyclosporin ( } n=23 \text { ) } \\
\text { Mean } \pm \text { SD (range) }\end{array}$ & $\begin{array}{c}\text { Placebo }(n=23) \\
\text { Mean } \pm \text { SD (range) }\end{array}$ \\
\hline Age (years) & $31 \cdot 6 \pm 12 \cdot 4(17-68)$ & $31 \cdot 0 \pm 9 \cdot 6(19-50)$ \\
\hline Race & $\begin{array}{l}22 \text { white Caucasians } \\
1 \text { Asian }\end{array}$ & $\begin{array}{l}22 \text { white Caucasians } \\
1 \text { Asian }\end{array}$ \\
\hline Sex & 11 females, 12 males & 12 females, 11 males \\
\hline Duration of disease (years) & $19 \cdot 8 \pm 13 \cdot 2(1-51)$ & $23 \cdot 7 \pm 13 \cdot 2(1-49)$ \\
\hline Number of relapses (previous year) & $3 \cdot 7 \pm 2 \cdot 0(1-10)$ & $4 \cdot 5 \pm 3 \cdot 3(1-12)$ \\
\hline Number of months free of lesions (previous year) & $1 \cdot 4 \pm 2 \cdot 1(0-6)$ & $1 \cdot 7 \pm 2 \cdot 3(0-6)$ \\
\hline
\end{tabular}

Table 3. Admission profile

they were taking drugs which were likely to interfere with the pharmacokinetics of cyclosporin. Appropriate contraceptive measures were imperative for women of child-bearing age. Pregnant or lactating women were excluded..$^{9.15 .16}$ The admission profile of the 46 patients included in this study is summarized in Table 3.

\section{Study medication}

Treatment with topical or systemic steroids or photochemotherapy was stopped 2 weeks before the study. Cyclosporin or placebo were administered as coded soft gelatine capsules of $25 \mathrm{mg}$ and $100 \mathrm{mg}$. Patients started on cyclosporin or placebo at a dose of $5 \mathrm{mg} / \mathrm{kg} /$ day. In the event of increases in creatinine, potassium, bilirubin, liver enzymes or blood pressure, the prescribed dose was decreased by $25-50 \%$.

Concomitant medication such as a systemic antihistamine (hydroxyzine 10 or $25 \mathrm{mg}$ ), and topical emollient therapy (white petrolatum) was prescribed throughout the study. However, in cases where the trial medication was observed to be effective, the use of an antihistamine and topical emollient was reduced or stopped as soon as possible. depending on the clinical condition of the patient.

Patients were to discontinue treatment prematurely in the event of side-effects and/or poor or no response [an improvement in 6-area total body severity assessment (TBSA) of $<20 \%$ ]. Patients who had either completed the trial, or had to discontinue treatment prematurely and had side-effects, were followed-up until all sideeffects had resolved. For laboratory parameters this was defined as a return to baseline.

\section{Clinical assessment}

For clinical assessment, variables such as the severity of the disease, lichenification, extent of the disease, itch. loss of sleep, and global efficacy were recorded.
The severity of the disease was assessed by trained physicians using a modified TBSA as described previously. ${ }^{11.12}$ Six regions were assessed, and included: (i) head and neck: (ii) a band across the trunk defined anatomically as lying ventrally between lines joining the apices of the anterior axillary folds and the inguinal ligaments and pubis, and dorsally between lines joining the inferior borders of the scapulae and the gluteal folds: (iii) the hands, including the wrists (ending at a line joining the lower ends of the radius and ulna); (iv) the feet, up to lines joining the medial and lateral malleoli; (v) the elbows, from mid-upper arms to mid-forearms: (vi) the knees, from mid-thighs to mid-calves. The most severely affected area in the specific region was scored.

Each region was assessed for erythema, infiltration. vesicles/papules, dryness/scaling, cracking/fissuring and excoriation/crusting. Each sign was graded for severity using the following scale: 0 , none: 1 , mild: 2 , moderate; 3 , severe.

Lichenification was scored separately following the same $0-3$ scale, and was not incorporated in the TBSA. ${ }^{9}$

The extent of the disease was assessed using the ruleof-nines area assessment' (RoNAA). ${ }^{9.11}$ Fractions of the individual regions (head and neck, ventral and dorsal trunk, left and right arm, genitalia, left and right leg) were defined in thirds. For example, the head and neck are equal to $9 \%$, and the area of atopic dermatitis may be $0,3.6$ or $9 \%$. The investigator indicated the area nearest to these four values.

At each assessment visit, itching and loss of sleep during the previous treatment period were scored, using the same scale $(0-3)$. The overall efficacy was scored by the patients and the investigators at week 6 , using the following scale: 0 , none: 1 , slight: 2 , moderate; 3 , good: 4 , very good.

Blood pressure, haematological and biochemical profiles, infection, use of drugs which influence renal function, or increase the risk of renal malfunction, were all monitored as described previously. ${ }^{11.16}$ 


\section{Statistics}

The statistical analysis of the results in all 46 patients was carried out using the SAS package (versions 6.06 and 6.07). All statistical tests were two-sided. A P-value of $\leqslant 0.05$ was considered as statistically significant. In the patients in whom treatment was stopped after 3 weeks the results were carried forward to week 6 . The two treatment groups were compared using the Wilcoxon rank-sum test. Fisher's exact test or the chisquared test were used in the case of categorial results. All patients were analysed according to the intention to treat' principle.

For the efficacy analysis, the baseline values of the several efficacy parameters were compared in the cyclosporin group and the placebo group using the Wilcoxon rank-sum test, in order to exclude possible differences at week 0 . The main endpoint was the TBSA. The RoNAA was also evaluated. The relative difference in the outcome of both assessments at week 6 was used in the analysis:

$$
100 \times \frac{\text { value at week } 6-\text { value at week } 0}{\text { value at week } 0} .
$$

The absolute difference between weeks 0 and 6 was also used for other variables such as the total score for lichenification, itching. loss of sleep, and the overall efficacy of the treatment as assessed by the patient and the investigator. The absolute difference was not computed for the last two variables.

Analysis of safety parameters (blood pressure, heart rate, body weight and laboratory parameters) was performed in the same manner, i.e. the difference between the values at weeks 0 and 6 was used in the outcome variable. Baseline values of several safety parameters in the cyclosporin group and in the placebo group were compared using the Wilcoxon rank-sum test, in order to exclude possible differences at week 0 .

\section{Results}

Fourteen patients on cyclosporin and nine on placebo completed the trial ( 6 weeks). Four patients on cyclosporin and 14 on placebo withdrew from the study because of lack of response to the trial medication (TBSA reduction to baseline of $<20 \%$ ). One patient on cyclosporin withdrew from the study because of hypertension which did not respond to a reduction in the drug dose. Four other patients in the cyclosporin group failed to complete the trial because of protocol violations. In all cases the last assessment prior to discontinuation was

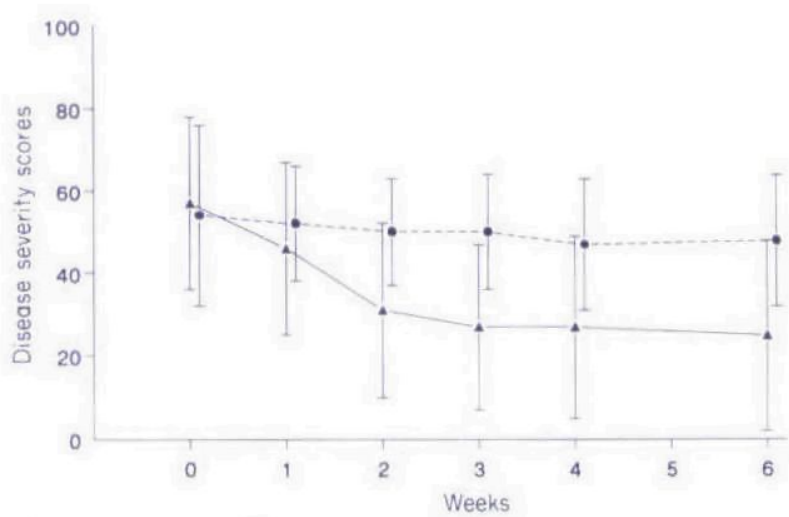

Figure 1. Scores of disease severity (6-area, total body severity assessment), mean values and standard deviations, in the cyclosporin and placebo groups. $\_$cyclosporin: - - placebo.

carried forward to all subsequent visits (intention to treat).

Mean scores of TBSA for each treatment group in time are shown in Table 1. There was a rapid decrease in the disease severity score over the first 2 weeks on cyclosporin therapy, and there was a mean improvement in disease severity score of $55 \%$ compared with baseline after 6 weeks (Table 1, Fig. 1). In the placebo-treated group, the mean percentage difference (week 0 vs. week 6) showed worsening (4\%). At week 6 , the difference between the cyclosporin group and the placebo group, expressed as the mean percentage shifts from the baseline values between the two groups, was statistically significant. Nine patients in the cyclosporin group who completed the trial had a significant response of $75 \%$ or more (percentage reduction of TBSA to baseline), and in three patients in this group $(n=14)$ this response was nearly $100 \%$. In the remaining five patients who

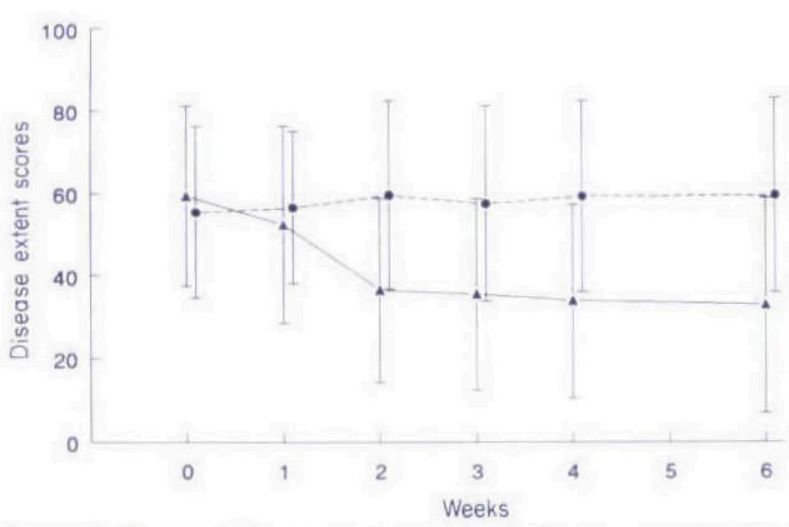

Figure 2. Scores of disease extent (rule-of-nines area assessment), mean values and standard deviations, in the cyclosporin and placebo groups. cyclosporin: - - placebo. 
Table 4. The overall efficacy at the end of cyclosporin and placebo treatment assessed by the patients and the investigators. (Score: 0 . none; 1 , slight: 2. moderate: 3 . good: 4 , very good)

\begin{tabular}{|c|c|c|c|c|c|c|}
\hline & 0 & 1 & 2 & 3 & 4 & Mean score \\
\hline Assessment by the investigators & $1(5 \cdot 3)$ & $3(15 \cdot 8)$ & $2(10 \cdot 5)$ & $5(26 \cdot 3)$ & $8(42 \cdot 1)$ & $2 \cdot 8 \pm 1 \cdot 3$ \\
\hline Assessment by the patients & $1(5 \cdot 3)$ & $3(15 \cdot 8)$ & $3(15 \cdot 8)$ & $5(26 \cdot 3)$ & $7(36 \cdot 8)$ & $2 \cdot 7 \pm 1 \cdot 3$ \\
\hline Assessment by the patients & $13(59 \cdot 1)$ & $3(13 \cdot 6)$ & $1(4 \cdot 5)$ & $3(13.6)$ & $2(9 \cdot 1)$ & $1 \cdot 0 \pm 1 \cdot 4$ \\
\hline$P$-value (Wilcoxon test) investigators & & & & & & $0.0001 \ddagger$ \\
\hline$P$-value (Wilcoxon test) patients & & & & & & $0 \cdot 0005 \div$ \\
\hline
\end{tabular}

$n$. number of patients.

*\% of cyclosporin group $(n=19)$;

$\dagger \%$ of placebo group $(n=22)$ :

$\doteqdot$ statistically significant.

completed the study the responses were $67,62,33,30$ and $26 \%$.

Lichenification improved gradually during cyclosporin therapy, from a mean score of 10.3 at baseline to 6.7 at week 6 ( $35 \%$ improvement). Improvement in the placebo group ranged from $10 \cdot 2$ at entry to $8 \cdot 8$ at week 6 (14\% improvement).

Mean scores of the RoNAA in each treatment group in relation to time are shown in Table 2 and Figure 2. There was a rapid improvement on cyclosporin during the first 2 weeks of treatment, and improvement continued until the end of treatment at week 6 . Overall, the mean improvement in the disease area in patients on cyclosporin was $40 \%$. No improvement in disease area was observed on placebo, and at week 6 the disease area had worsened by a mean of $25 \%$. After 6 weeks of therapy. the difference between the two groups, expressed as the mean percentage shifts of mean values, was statistically highly significant (Table 2).

At week 6 , the mean scores of itch in the cyclosporintreated group had improved from $2 \cdot 7$ to $1 \cdot 1$, compared with an improvement from $2 \cdot 6$ to $2 \cdot 1$ in the placebo group. The difference between the two groups in the improvement of itch was statistically significant $(P=0.01)$. This apparently resulted in an improvement in sleep in the cyclosporin group. The difference between the two groups in the improvement in sleep was also statistically significant $(P=0 \cdot 001)$.

Twelve patients on cyclosporin ( $52 \%$ ) reported a total of 28 adverse events, compared with five patients ( $22 \%)$ on placebo who reported a total of nine adverse events. Of the 28 adverse events in patients on cyclosporin, 15 were thought to have a probable relationship to therapy, and five were thought to have a definite relationship to therapy. Three patients on cyclosporin experienced severe adverse events (one patient had nausea, and two developed hypertension), compared with three patients on placebo (one patient had pain in the legs and was feverish, one had a swollen face and eyelids. and another patient was dyspnoeic).

The overall efficacy at the end of treatment with cyclosporin and placebo, as assessed by the patients and the investigators, is shown in Table 4. Differences between the cyclosporin and the placebo groups were statistically highly significant. Tolerability of cyclosporin and placebo was rated as good or very good by the majority of the patients and the investigators.

At week 6, no statistically significant differences in any of the haematological and biochemical parameters were observed between the cyclosporin and placebo groups, apart from the eosinophil count $(P=0 \cdot 008)$.

The difference between serum creatinine values at week 6 (mean increase $3 \cdot 5 \pm 15 \mu \mathrm{mol} / \mathrm{l}$ ) and baseline (mean value $74.5 \pm 12.5 \mu \mathrm{mol} / \mathrm{l}$ ) in the cyclosporin group, compared with the difference in the placebo group, was statistically significant $(P=0 \cdot 02)$. A decrease in the creatinine clearance in the cyclosporin group (mean decrease $4 \cdot 2 \pm 20$ ), compared with the baseline (mean value $111 \pm 19$ ), was in agreement with the rise in serum creatinine in this group. An increase in the 
bilirubin (mean increase $3 \cdot 4 \pm 5 \cdot 7 \mu \mathrm{mol} / \mathrm{l}$ ), but not in the other biochemical parameters of liver function, was observed in the cyclosporin group.

\section{Discussion}

This study corroborates the findings of previous studies $^{2-12}$ that short-term treatment of severe chronic atopic dermatitis with cyclosporin is effective and safe. Compared with the placebo, patients on cyclosporin showed statistically significant subjective and objective improvements in disease severity score (TBSA), lichenification score, disease extent score (RoNAA), itch, loss of sleep and overall efficacy at the end of the 6-week treatment period. The superiority of cyclosporin over placebo as measured by TBSA and RoNAA was further supported by the overall efficacy assessments.

It is important to note that the objective clinical features used for the assessment of the disease severity (TBSA) differ from those used in other studies, ${ }^{11,12.17}$ Costa et al. ${ }^{17}$ assessed erythema, oedema, vesicles, crusts. excoriations, scaling, lichenification. and pigmentary changes. Other trials have employed scores of erythema. purulence, excoriation or crusting, dryness or scaling, cracking or fissuring, and lichenification. ${ }^{11.12}$

In contrast with previous studies ${ }^{9}$ we assessed lichenification of the skin, which is apparently more resistant to short-term cyclosporin therapy. ${ }^{8}$ separately from the disease activity (TBSA). All the different parameters were graded separately in the most severely affected area in the specified region (TBSA).

The differences in the clinical parameters contributing to the disease severity score (TBSA) could be an explanation for discrepancies in the values for (mean) clinical improvement in the present and other studies. ${ }^{11}$ The use of the final assessments, following the 'intention to treat' principle, in patients who discontinue the trial, may influence the mean values for efficacy (at week 6 ) in an unfavourable way, and this might be another explanation. However, 10 patients in the cyclosporin group $(n=14)$. who completed the study, had a significant beneficial response to this drug at week 6 . The efficacy of cyclosporin was also emphasized by the number of patients on placebo who withdrew from the study (14/23 compared with 4/23 in the cyclosporin group).

Creatinine and bilirubin values which were increased on the last day of the trial returned to baseline within 8 weeks during the post-treatment observation period. However, one patient, in whom the final bilirubin value during cyclosporin therapy was slightly above normal, could not be evaluated at follow-up. In the two patients with hypertension. the blood pressure returned to normal levels during post-treatment observation.

The time to relapse of the disease could not be determined in this study. The possibility of a rapid relapse in severe psoriasis after withdrawal of cyclosporin is well documented. ${ }^{15.16}$ In a recent randomized, double-blind, controlled crossover study, relapse of atopic dermatitis was rapid after stopping cyclosporin, but the mean scores for disease activity and extent of disease were less than the baseline values. However, there was no relapse in the overall quality of life scores 8 weeks after cyclosporin was withdrawn. ${ }^{12}$

Maintenance therapy with cyclosporin for atopic dermatitis is effective, but controversy with regard to the minimum effective dose, and exacerbation of the disease after discontinuation of treatment, ${ }^{11.18}$ suggests a need for further studies.

Cyclosporin has been shown to reduce the numbers of helper/inducer $\mathrm{T}$ cells and the number of activated cells expressing interleukin- 2 receptor $\left(\amalg-2 \mathrm{R}^{+}\right),{ }^{9,19}$ and these changes correlated with a marked improvement in the disease, supporting the view that atopic dermatitis is based on T-cell-mediated inflammation.

Although other authors have confirmed the relative safety of cyclosporin in paediatric organ transplantation, ${ }^{20}$ we recommend that further studies on the use of cyclosporin in the treatment of atopic dermatitis in children should be undertaken. Short-term treatment with cyclosporin has beneficial effects on the quality of life in severe atopic dermatitis in terms of crisis intervention. ${ }^{1.11 .12}$

In conclusion, oral cyclosporin at a dose of $5 \mathrm{mg} / \mathrm{kg} /$ day for 6 weeks is an effective, safe, and well-tolerated treatment for atopic dermatitis in adults. Cyclosporin can significantly improve the acute or subacute disease severity, the extent of the disease, the lichenification, itch, and loss of sleep. Provided that the patients are carefully monitored according to suggested guidelines, ${ }^{21}$ short-term (approximately 2 months) therapy with cyclosporin can be safely used during severe exacerbations of atopic dermatitis.

\section{Acknowledgments}

We thank Dr H.J.J.van Lier (Department of Medical Statistics, University of Nijmegen), Mr J.C.J.N.Stokvis (Sandoz BV, the Netherlands) and Dr B.Tank (Erasmus University, Rotterdam) for valuable suggestions. 


\section{References}

1 Champion RH. Parish WE. Atopic dermatitis. In: Textbook of Dermatology (Champion RH. Burton JL. Ebling FJG, eds), 5 th edn.. Vol. 1. Oxford: Blackwell Scientific Publications. 1992: 589-610.

2 Van Joost Th. Stolz E. Heule F. Efficacy of low-dose cyclosporine in severe atopic skin disease. Arch Dermatol 1987: 123: 166-7.

3 Logan RA. Camp RDR. Severe atopic eczema: response to oral cyclosporin A. I R Soc Med 1988: 81: 417-18.

4 Motley RJ. Whittaker JA. Holt PJA. Resolution of atopic dermatitis in a patient treated with cyclosporin. Clin Exp Dermatol 1989: 14: 243-4.

5 Taylor RS 3d, Cooper KD, Headington IT et al. Cyclosporine therapy for severe atopic dermatitis. / Am Acad Dermatol 1989: 21: $580-3$.

6 Gold MH. Picascia DD, Roenigk HH Jr. Treatment-resistant atopic dermatitis controlled with cyclosporin A. Int J Dermatol 1989:28: 481-2,

7 Ross JS. Camp RDR. Cyclosporin $\mathrm{A}$ in atopic dermatitis. $\mathrm{Br} /$ Dermatol 1990: 122(Suppl. 36): 41-5.

8 Munro CS. Higgins EM. Marks JM et al. Cyclosporin A in atopic dermatitis: therapeutic response is dissociated from effects on allergic reactions. Br J Dermatol 1991: 124: 43-8.

9 Van Joost Th. Kozel MMA. Tank B et al. Cyclosporine in atopic dermatitis. Modulation in the expression of immunologic markers in lesional skin. I Am Acad Dermatol 1992: 27: 922-8.

10 Korstanje MJ. Van de Staak WJBM. Cyclosporin maintenance therapy for severe atopic dermatitis. Acta Derm Venereol (Stockh) 1991: $71: 356-7$.
11 Sowden JM. Berth-Jones J. Ross JS et al. Double-blind, controlled. crossover study of cyclosporin in adults with severe refractory atopic dermatitis. Lancet 1991: 338: 137-40.

12 Salek MS, Finlay AY, Luscombe DK et al. Cyclosporin greatly improves the quality of life of adults with severe atopic dermatitis. A randomized. double-blind placebo-controlled trial. $\mathrm{Br}$ J Dermatol 1993: 129: 422-30.

13 Heule F, Laeijendecker R, van Joost Th. Topical cyclosporin A treatment in psoriasis and other dermatological diseases: theoretical and practical aspects. / Dermatol Treat 1992: 2: 149-53.

14 Hanifin JM. Rajka G. Diagnostic features of atopic dermatitis. Acta Derm Venereol (Stockh) 1980: 92(Suppl. 144): 44-7.

15 Van Joost Th, Bos JD. Heule F. Meinardi MMHM. Low-dose cyclosporin $\mathrm{A}$ in severe psoriasis. A double-blind study. $\mathrm{Br} /$ Dermatol 1988: 118: 183-90.

16 Bos JD. Meinardi MMHM. van Joost Thet al. Use of cyclosporin in psoriasis. Lancet 1989: ii: 1500-2.

17 Costa C. Rillet A. Nicolet M. Saurat JH. Scoring atopic dermatitis: the simpler the better? Acta Derm Venereol (Stockh) 1989; 69: $41-5$.

18 Sepp N. Fritsch PO. Can cyclosporin A induce permanent remission of atopic dermatitis? Br / Dermatol 1993: 128: 213-16.

19 De Prost Y. Management of severe atopic dermatitis. Acta Derm Venereol (Stockh) 1992:(Suppl. 176): 117-19.

20 Keown PA. Lirenman D. Landsberg D et al. Use of cyclosporin in pediatric transplantation. Immunology, pharmacology and therapeutic implications. Clin Transpl 1991: 5: 181-5.

21 Camp RDR, Reitamo S. Friedman PS et al. Cyclosporin A in severe. therapy-resistant atopic dermatitis: report of an international workshop, April 1993. Br / Dermatol 1993: 129: 217-20. 
This document is a scanned copy of a printed document. No warranty is given about the accuracy of the copy. Users should refer to the original published version of the material. 